\title{
Effect of incident angle on ultrasonic transmission in steam flow for use with clamp-on ultrasonic flowmeter
}

\author{
Hideki MURAKAWA*, Shuhei ICHIMURA*, Masaki SHIMADA*, Katsumi SUGIMOTO*, \\ Hitoshi ASANO*, Shuichi UMEZAWA** and Katsuhiko SUGITA** \\ * Graduate School of Engineering, Kobe University \\ 1-1 Rokkodai, Nada, Kobe 657-8501, Japan \\ E-mail: murakawa@mech.kobe-u.ac.jp \\ ** Tokyo Electric Power Company Holdings, Inc. \\ 4-1 Egasaki, Tsurumi, Yokohama 230-8510, Japan
}

Received: 9 March 2020; Revised: 13 April 2020; Accepted: 23 April 2020

\begin{abstract}
Energy management in industrial plants requires measurements of the steam flow rates at each usage location. A clamp-on ultrasonic flowmeter can be used to effectively measure the steam flow rates in existing pipes. An ultrasonic flowmeter is used to calculate the transit time of ultrasonic signals between the downstream and upstream sensors, which is affected by the line-averaged velocity along the ultrasonic beam. The intensity of the transmitted ultrasonic signal is crucial for measuring the steam flow rate using the clamp-on ultrasonic flowmeter. The authors focused on the effects of the ultrasonic incident angle on the transmitted ultrasonic signal intensity in steam flow. Ultrasonic transmission experiments were carried out on three pipes (SGP 25A, SGP 50A and SGP 80A) filled with stationary nitrogen, and the transit time in steam flow was measured on SGP $80 \mathrm{~A}$ pipe by changing the ultrasonic incident angle. The pipes are made of carbon steel and generally used for the steam flow. The results indicate that the appropriate incident angle that allowed the maximum transmitted signal intensity differed depending on the thickness of the pipe wall. Furthermore, a good agreement with the critical angles of the zero-order of symmetric mode in Lamb waves was noted. Thus, the propagated ultrasonic waves can be considered Lamb waves, which increase signal intensity. The transmitted signal intensity decreases because of the turbulent dissipation as the steam velocity increases. Appropriately setting the incident angle depending on the wall thickness, particularly for higher steam flow rates, is essential for evaluating the transit time difference between upstream and downstream transducers.
\end{abstract}

Keywords : Steam flow rate, Clamp-on, Ultrasonic flowmeter, Time-of-flight, Incident angle, Lamb wave

\section{Introduction}

The ratio of heat demand to energy supply is high in many industrial plants. Most of the heat demand is supplied by steam. The saturated steam is generated in boilers and supplied to usage places through a piping system. The steam supply pipes must often feed a long distance. Therefore, condensation may occur as the steam flows through the piping system due to heat loss. Even though the steam flow rate is controlled at the boiler level, the actual steam flow rates at different consumption points may vary. The optimize of energy management requires steam flow rate measurements at each such instance. However, steam flowmeters are usually not installed in many cases. Orifice, vortex and inline ultrasonic flowmeters have been widely used to measure the steam flow rates (Steven et al., 2009; Umezawa et al., 2013). They operate across a wide range of flow rates but must be connected to the pipes. It is difficult to install them in existing pipes, which makes clamp-on ultrasonic flowmeters an attractive solution.

Ultrasonic flowmeters have been developed for more than 60 years and these instruments are widely used to measure the flow rates in liquid and gas flows (Lynnworth and Liu, 2006). Most ultrasonic flowmeter technology is based on the time-of-flight (TOF) of ultrasonic pulses. Figure 1 shows the schematic of the TOF ultrasonic flowmeter. 
Two ultrasonic transducers are set on the outer surface of the test pipe at upstream and downstream positions. Ultrasonic pulses are emitted at the upstream ultrasonic transducer, and the pulses are received at the downstream transducer and vice versa. The transit time of the ultrasonic signal between the downstream and upstream transducers is then calculated. The TOF is influenced by the line-averaged velocity, $V_{\mathrm{L}}$, along the ultrasonic beam. The TOF is influenced not only by $V_{\mathrm{L}}$ but also by the speed of sound of the fluid. It is possible to neglect the slight change in the speed of sound in the fluid by using the transit time difference, $\Delta t$, to calculate $V_{\mathrm{L}}$ for the ultrasonic flowmeter, as follows:

$$
\Delta t=t_{2}-t_{1}=\frac{2 L V_{L} \cos \theta}{C^{2}-V_{L}^{2} \cos ^{2} \theta}
$$

where $\theta$ is the refract angle into the flow, $C$ is the sound speed, $L$ is the propagation distance of the ultrasonic wave. $t_{1}$ and $t_{2}$ represent the transit time from upstream and downstream transducers, respectively. The calculation of the flow rate, $Q$, from the $V_{\mathrm{L}}$, profile factor, $P F$, is:

$$
Q=\frac{\pi D^{2}}{4} \cdot V=P F \cdot \frac{\pi D^{2}}{4} \cdot V_{\mathrm{L}}
$$

where $D$ is the inner diameter of the pipe. $P F$ is a conversion factor from $V_{\mathrm{L}}$ to area-averaged velocity, $V$, and depends on the velocity profiles. It is generally determined on factors such as the calibration considering the flow rate, and upstream and downstream conditions. If the velocity profile, $v(r)$, in a pipe can be considered as power-law given as

$$
\frac{v(r)}{v_{\max }}=\left(1-\frac{r}{R}\right)^{\frac{1}{n}}
$$

the $P F$ can be expressed as

$$
P F=\frac{2 n}{2 n+1}
$$

where $r$ is the distance from the pipe center, $R$ is the pipe radius, and the $v_{\max }$ is the maximum velocity at the pipe center. The accurate measurement of $\Delta t$ is important for the calculation of $V_{\mathrm{L}}$ to evaluate the flow rate with the TOF ultrasonic flowmeter.

Many studies have been carried out to improve gas and steam flow rate measurements using ultrasonic flowmeters. Morita et al. (2017) measured the flow rate of wet steam with an ultrasonic flowmeter and they observed that the flow rate error increased with wetness and high flow velocity. They used a commercial ultrasonic flowmeter where the sensors were in direct contact with the flow field. Ramadas et al. (2016) investigated the wetness effect on gas flow measurements. Kurniadi and Trisnobudi (2006) developed a method to measure the two-dimensional velocity profiles of gas flows using ultrasonic tomography based on the transit time. Evaluation method of steam quality using a TOF flowmeter was proposed by Shen (1999). However, these studies are focused on in-line ultrasonic flowmeters.

Although the clamp-on type ultrasonic flowmeter has the potential to measure the steam flow rate, the intensity of the transmitted ultrasound signal becomes weak in gas flows because the signal attenuation is more pronounced in gas rather than in liquid. Additionally, the significant acoustic impedance difference between the pipe material and gas-phase contributes to the lower intensity of the transmitted ultrasonic signal. Therefore, the signal-to-noise ratio (SNR) for gas flow measurements decreases in comparison to that for liquid flow measurement. Although there are some ultrasonic flowmeters for gas flow rates, most are in-line flowmeter. In addition to the above issues, the high temperature of the pipe and wetness in the steam are also critical factors. The sensitivity of the piezoelectric elements in the sensors decreases at higher temperatures. Furthermore, the wetness of the steam varies due to heat loss, which 
affects the characteristics of the ultrasonic signal propagation. These factors pose a significant challenge for obtaining accurate steam flow rate measurements using clamp-on ultrasonic flowmeters. As a result, only a few ultrasonic flowmeters can be employed for steam but the applicable conditions are limited. Therefore, it is important to improve the SNR of the measurements.

There are a few studies on measuring the gas and steam flow rates using clamp-on ultrasonic flowmeters. Nishiguchi et al. (2016) developed an ultrasonic transducer with a curved polystyrene wedge to improve the SNR for accurate measurements of the gas flow rate at low-pressure conditions $(<0.06 \mathrm{MPa})$. Murakawa et al. (2020) developed a method consulting standard deviation to calculate the transit time difference under low SNR conditions in two-phase flow. Furthermore, the presence of liquid film on the inside of the pipe wall increased the noise. Sasaki et al. (2017) developed a damping material to improve the SNR for steam flow measurement using a clamp-on ultrasonic flowmeter. Fan et al. (2018) proposed to use a leaky Lamb waves for air-coupled gas flowmeters. Sasaki and Hayashi (2015) investigated the transmitted ultrasonic intensity on a stainless pipe (outer diameter of $41.2 \mathrm{~mm}$, wall thickness of 3.7 $\mathrm{mm}$ ) filled with city gas. They examined three incident angles of 54,57 , and $60^{\circ}$, with the angle of $57^{\circ}$ yielding the best SNR results. They assumed that the ultrasonic transmitted from the pipe wall to the gas as an evanescent wave. However, the appropriate incident angle has not been fully understood to predict it for each steam flow pipe.

The purpose of this study is the evaluation of the appropriate incident angle of the ultrasonic sensor for clamp-on ultrasonic flowmeter in measuring the steam flow rate to improve the SNR. Transmitted ultrasonic intensities were measured by changing the ultrasonic incident angle with carbon steel pipes that are generally used for the steam flow. Furthermore, transit time differences in steam flow were determined. We then discussed the appropriate incident angles in each pipe and the associated ultrasonic propagation mode.

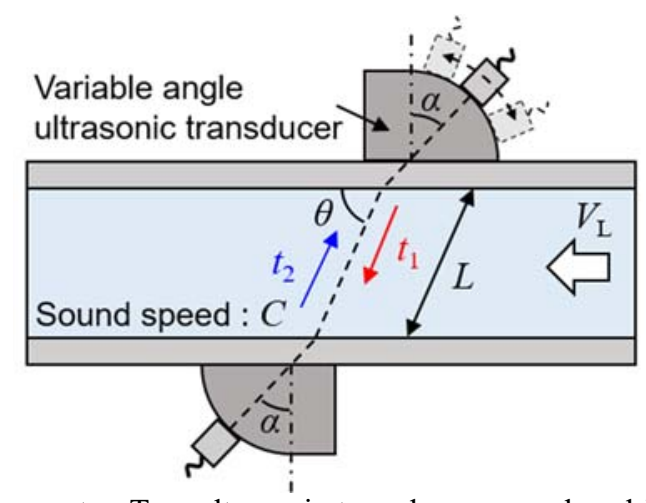

Fig. 1 Schematic of TOF ultrasonic flowmeter. Two ultrasonic transducers are placed in upstream and downstream positions. Ultrasound pulses are emitted, and the signal is received at the other side of the transducer. Based on the transit time, the line-average velocity of the flow is evaluated.

\section{Experimental setup and methods}

Two experiments to better understand ultrasonic transmission were carried out. The first experiment was conducted on pipes filled with nitrogen. Changes of intensities of ultrasonic transmission in each selected contact angle were examined on three different carbon steel pipes and different system pressures. The second experiments involved steam flow measurements in each contact angle to clarify the relation between the transmitted intensity and flow.

\subsection{Ultrasound transmission in differently sized pipes filled with nitrogen}

The appropriate incident angle of the ultrasonic was examined through ultrasonic transmission experiments in three pipes of different sizes, filled with nitrogen. The schematic of the experimental setup is shown in Fig. 2. A silicon rubber with mixed materials developed by Sasaki et al. (2017) was used as a damping material of ultrasonic propagation inside the pipe referred to as guided waves. The rubber was set on the pipe wall to attenuate the guided 
waves. Variable angle ultrasonic transducers (model: 1C30×10VA, Japan Probe Co. Ltd., Japan) that can continuously change the incident angle of ultrasound to the pipe wall, $\alpha$, between $0-65^{\circ}$, were used for the ultrasonic transmission and the reception as shown in Fig. 1. The wedge material was polyetherimide. Two sensors were horizontally set at the outer wall of the test section facing each other. The incident angle was set at the same value for both transducers. Experiments were carried out with three pipes (SGP 25A, SGP 50A and SGP 80A) of different diameters made of carbon steel to evaluate the influence of the pipe diameter on the target signal intensity. The pipes were black pipes without coating. These pipes are commonly used for the transport of steam. Their specifications are listed in Table 1. The experimental conditions are listed in Table 2. Ultrasonic reflection and transmission intensities are influenced by the difference of the acoustic impedance of the material. The absolute pressures of nitrogen were selected at 0.45 and $0.7 \mathrm{MPa}$ because acoustic impedances of nitrogen at $0.45 \mathrm{MPa}$ and saturated steam at $0.7 \mathrm{MPa}$ are almost the same as shown in Table 3.

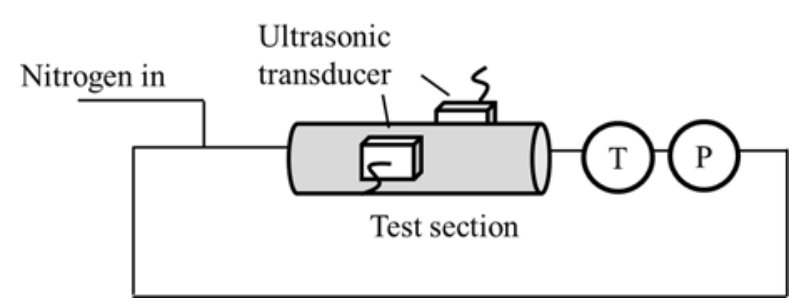

Fig. 2 Schematic of the experimental setup for ultrasonic transmission experiments. Pipes are filled with stationary nitrogen. The experiment was carried out with different pipes and pressures.

Table 1 Geometry of the carbon steel pipes.

\begin{tabular}{l|c|c|c}
\hline \hline & SGP 25A & SGP 50A & SGP 80A \\
\hline Inner diameter & $27.6 \mathrm{~mm}$ & $52.9 \mathrm{~mm}$ & $80.7 \mathrm{~mm}$ \\
\hline Wall thickness & $3.2 \mathrm{~mm}$ & $3.8 \mathrm{~mm}$ & $4.2 \mathrm{~mm}$ \\
\hline
\end{tabular}

Table 2 Experimental conditions at stationary nitrogen using variable angle sensors.

\begin{tabular}{c|c}
\hline \hline Incident angle & $45 \sim 60^{\circ}$ \\
\hline Pressure & $0.45 \mathrm{MPa}, 0.7 \mathrm{MPa}$ \\
\hline Temperature & $16 \sim 17^{\circ} \mathrm{C}$ \\
\hline
\end{tabular}

Table 3 Physical properties of nitrogen and saturated steam (REFPLOP, 2018).

\begin{tabular}{c|c|c|c|c|c}
\hline \hline Substance & Pressure, $P$ & Temperature, $T$ & Sound speed, $C$ & Density, $\rho$ & Acoustic impedance, $Z=\rho \cdot C$ \\
\hline Nitrogen & $0.45 \mathrm{MPa}$ & $20^{\circ} \mathrm{C}$ & $349.6 \mathrm{~m} / \mathrm{s}$ & $5.18 \mathrm{~kg} / \mathrm{m}^{3}$ & $1.81 \times 10^{3} \mathrm{~kg} /\left(\mathrm{m}^{2} \cdot \mathrm{s}\right)$ \\
\hline Nitrogen & $0.70 \mathrm{MPa}$ & $20^{\circ} \mathrm{C}$ & $350.0 \mathrm{~m} / \mathrm{s}$ & $8.06 \mathrm{~kg} / \mathrm{m}^{3}$ & $2.82 \times 10^{3} \mathrm{~kg} /\left(\mathrm{m}^{2} \cdot \mathrm{s}\right)$ \\
\hline Saturated steam & $0.70 \mathrm{MPa}$ & $165.0^{\circ} \mathrm{C}$ & $497.6 \mathrm{~m} / \mathrm{s}$ & $3.67 \mathrm{~kg} / \mathrm{m}^{3}$ & $1.82 \times 10^{3} \mathrm{~kg} /\left(\mathrm{m}^{2} \cdot \mathrm{s}\right)$ \\
\hline
\end{tabular}

\subsection{Steam flow measurement}

We used the same steam flow loop as Umezawa et al. (2013) and Morita et al. (2017) for our steam flow experiments. The respective experimental setup is shown in Fig. 3. It consists of a heat exchanger, boiler (model: AI-2000 25H, Miura Co. Ltd., Japan), heater, cooling coil, water storage tank, test section, Coriolis flowmeter, pressure gauge, and thermometer. Saturated steam is supplied from the boiler, and the steam wetness was controlled using the heater and cooling coil. The steam was circulated through the test section. The system pressure and the flow rate were controlled with the pressure control and the back pressure valves set at upstream and downstream of the test section. The test section was the SGP 80A black pipe without coating. The ultrasonic transducers and damping material were the same with the ultrasonic transmission experiments at a pipe filled with nitrogen. The transducers were installed 
horizontally and facing each other. The experimental conditions are shown in Table 4 . The system pressure was set at $0.7 \mathrm{MPa}$ with saturation temperature of $165^{\circ} \mathrm{C}$. The degree of super heat was between 3 and $8{ }^{\circ} \mathrm{C}$. The steam flow velocity was set to three conditions. The extended uncertainty of the steam flow loop was evaluated at $0.61 \%$ (coverage factor $k=2$ ) reported by Morita et al. (2017).

Table 4 Experimental conditions for SGP 80A in steam flow.

\begin{tabular}{c|c}
\hline \hline Incident angle & $48 \sim 60^{\circ}$ \\
\hline Pressure & $0.7 \mathrm{MPa}$ \\
\hline Temperature & $168 \sim 173^{\circ} \mathrm{C}$ \\
\hline Area-averaged steam flow velocity & $5.8,12,20 \mathrm{~m} / \mathrm{s}$ \\
\hline
\end{tabular}

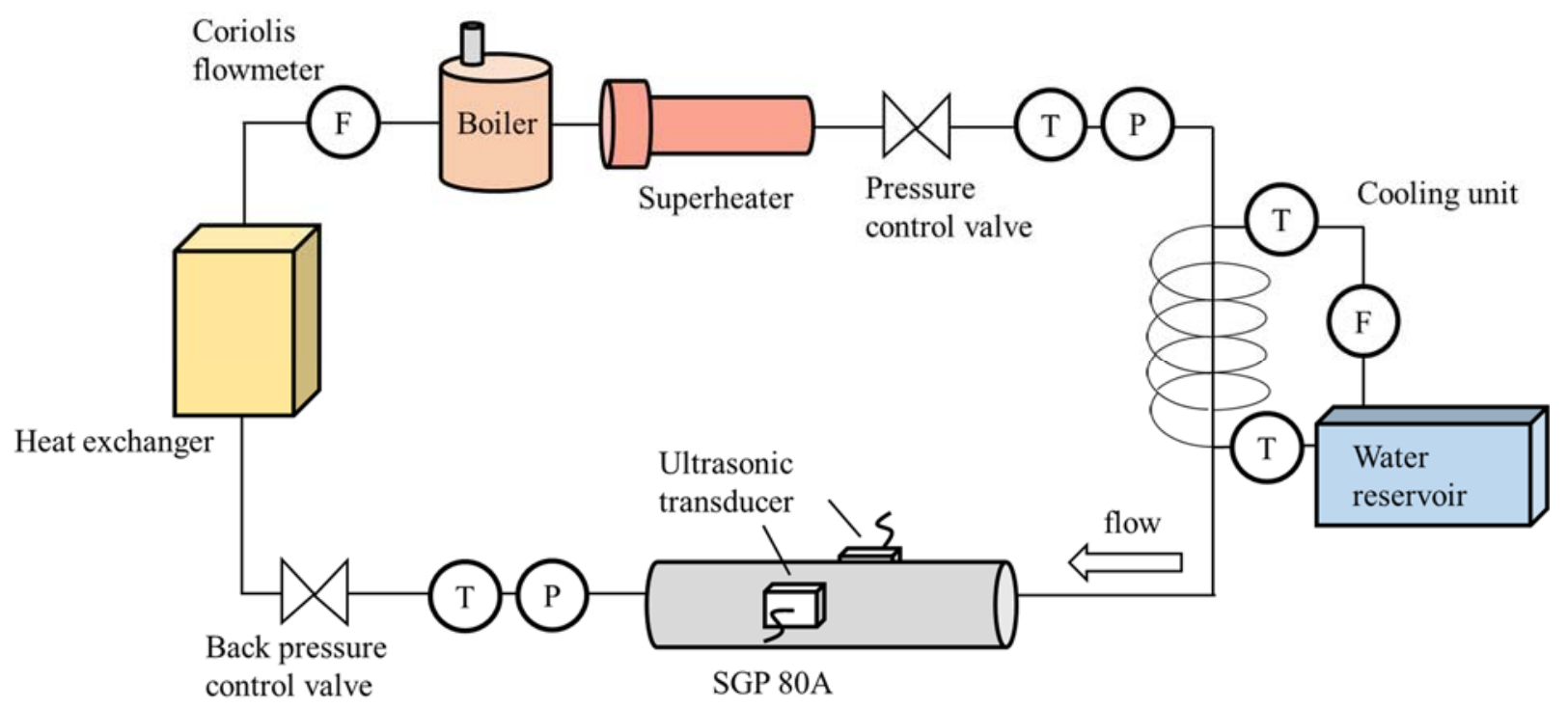

Fig. 3 Schematic of the experimental setup of the steam flow measurement. Superheated steam was supplied to the horizontal test section at $0.7 \mathrm{MPa}$ in the SGP $80 \mathrm{~A}$ pipe. The variable angle ultrasonic transducers were set facing each other horizontally.

\subsection{Measurement system and the procedure}

The ultrasonic transducers were connected to a pulser/receiver (model: JPR-10C-2CH-KB, Japan Probe Co. Ltd., Japan). Four-cycle burst signals at $1 \mathrm{MHz}$ were emitted as the ultrasonic signals. Both of the ultrasonic transducers had the same center frequency of $1 \mathrm{MHz}$. The ultrasonic pulses were periodically emitted from the transducers at a pulse repetition frequency of $100 \mathrm{~Hz}$. The ultrasonic signals were received at the opposite transducer and sampled using a high-speed digitizer (model: NI PXI-5114, National Instruments Corporation) with a sampling rate of $250 \mathrm{MS} / \mathrm{s}$ (mega-samples per second). Ultrasonic signals were continuously recorded in each direction: from the upstream transducer to the downstream transducer and vice versa. Using the consecutive signals, the ensemble-averaged signals were calculated in each direction.

\section{Results}

\subsection{Ultrasonic transmission at a pipe filled with nitrogen}

Fig. 4 shows an example of the ensemble averaged transmitted ultrasonic signal in stationary nitrogen at $0.7 \mathrm{MPa}$. The incident angle was set at $54^{\circ}$. The horizontal axis represents the elapsed time from the ultrasonic emission, $t$. The 
vertical axis represents the amplitude of the received signal. The ultrasonic signal transmitted through the stationary nitrogen was clearly observed at approximately $285 \mu \mathrm{s}$, which was the target signal for the measurements. The high signal intensities at the beginning of the elapsed time $(t<5 \mu \mathrm{s})$ represented the noise associated with the burst signals of the emitted pulse because the ultrasonic sensors were connected with the same pulser/receiver for the pulse emission and the reception. Except for the burst signal, high voltage signals cannot be confirmed until $75 \mu$ s; signals between 75 $\mu \mathrm{s}<t<285 \mu \mathrm{s}$ represented the propagated signals in the pipe wall, i.e., guided wave. A partial ultrasonic signal from the outer surface of the pipe entered the nitrogen, but the remainder reflected on the inner surface of the pipe. As the speed of sound in the carbon steel is higher than in the nitrogen, the ultrasound propagated in the pipe wall and appeared as early noise. Peak-to-peak intensity of the target signal was calculated for each incident angle based on recordings.

The relationship between the incident angle and the signal intensity in each pipe is shown in Fig. 5. The horizontal axis represents the incident angle of the ultrasound, and the vertical axis represents the signal intensity normalized by the maximum values in each pipe and pressure condition. The transmitted signal intensities changed with the incident angle. The maximum intensities were confirmed at the incident angle of $47-48^{\circ}$ for SGP $25 \mathrm{~A}$ pipe, $50^{\circ}$ for $50 \mathrm{~A}$ pipe, and $54^{\circ}$ for $80 \mathrm{~A}$ pipe. The tendency is almost the same at $P=0.45$ and $0.7 \mathrm{MPa}$. Thus, the incident angles that allowed the maximum intensity were considered as the appropriate incident angle for better SNR, and they depended on the pipe size. The appropriate incident angle increased with the pipe diameter that changes the pipe wall thickness.

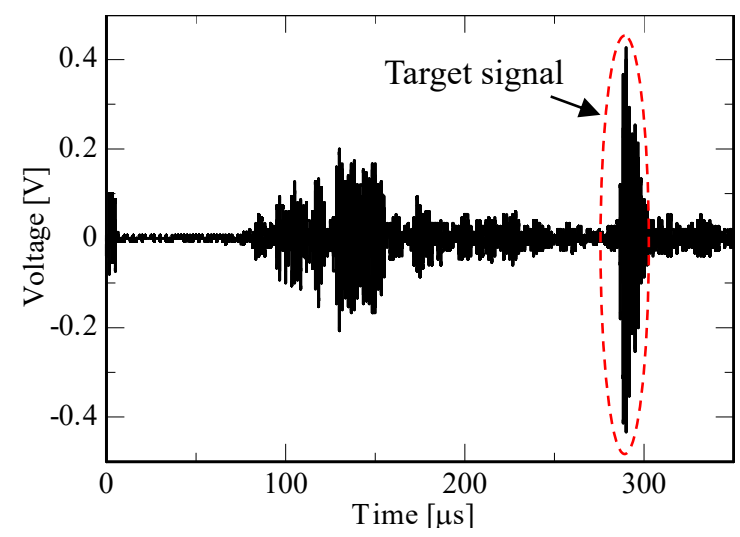

Fig. 4 Received ultrasonic signals for SGP 80A pipe filled with nitrogen at $0.7 \mathrm{MPa}$. The target signal that propagated in nitrogen was clearly confirmed at approximately $285 \mu \mathrm{s}$ as indicated by the red dashed line. The signals prior to the target signal were guided waves that propagated inside the pipe wall.

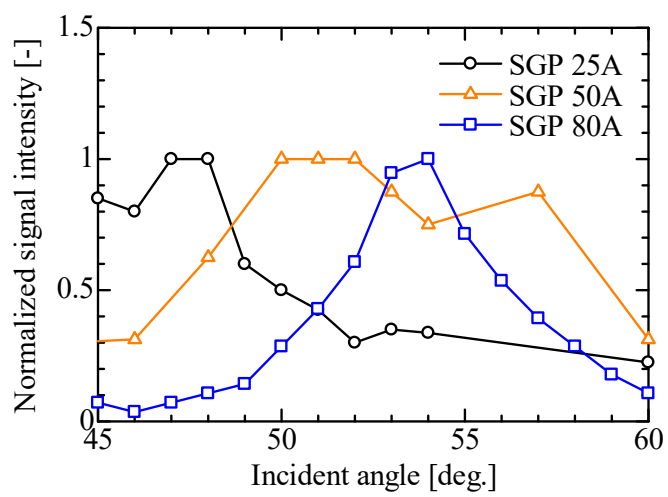

(a) $P=0.45 \mathrm{MPa}$

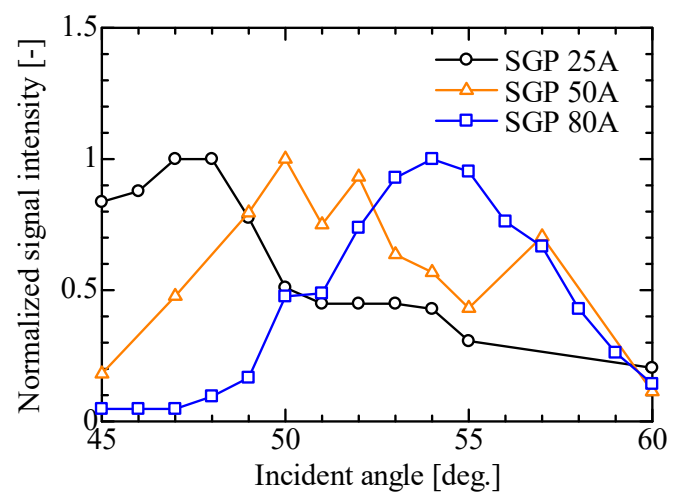

(b) $P=0.7 \mathrm{MPa}$

Fig. 5 Relationship between incident angle and maximum voltage of the target signal at pipes filled with nitrogen. Differences of color and the symbol represent the difference of the pipe diameter. The distributions depend on the pipe size. 


\subsection{Steam flow measurement}

The relationship between the incident angle and the signal intensity for the 80A pipe in steam flow is shown in Fig. 6. The signal intensity is normalized by the maximum intensity at $V=5.8 \mathrm{~m} / \mathrm{s}$. The area-averaged steam flow velocity spanned 5.8 to $20 \mathrm{~m} / \mathrm{s}$, which corresponds to a Reynolds number of $1.2 \times 10^{5}$ to $4.0 \times 10^{5}$. The intensity of the target signal was largest at an incident angle of $54^{\circ}$ for all velocities in this experiment. The target signal intensity decreased as the flow velocity increased because the turbulent intensity increased as the velocity increased. The diffusion of the ultrasonic wave in the fluid increased with the fluid velocity resulting in a weaker signal.

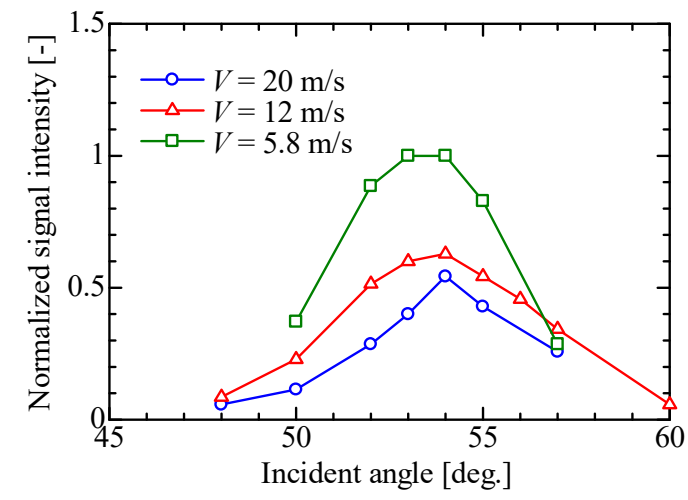

Fig. 6 Relationship between incident angle and normalized signal intensity in steam flow (80A pipe). The differences of color and symbols represent the area-averaged steam flow velocity. Slowest velocity yielded highest amplitude.

Fig. 7 shows the ensemble-averaged ultrasonic-signals transmitted from the upstream and downstream ultrasonic transducers in steam flow at $V=12 \mathrm{~m} / \mathrm{s}$. The target signals were confirmed at approximately $220 \mu \mathrm{s}$. The time difference in the target signal between nitrogen and the steam flow was caused by the difference in the material-specific speed of sound. Furthermore, the up- to downstream signals appear slightly early than that from down- to upstream. From the averaged wave signal, the transit time difference between the upstream and downstream ultrasonic transducers, $\Delta t$, was estimated using the normalized cross-correlation function, $R(\tau)$ :

$$
R(\tau)=\frac{\int E_{\mathrm{U}}(t) E_{\mathrm{D}}(t+\tau) d t}{\sqrt{\int E_{\mathrm{U}}^{2}(t) d t \int E_{\mathrm{D}}^{2}(t+\tau) d t}}
$$

where the $E_{\mathrm{U}}$ and $E_{\mathrm{D}}$ represent the signals received from the upstream and downstream ultrasonic transducers, respectively. The value of $\tau$ at which $R(\tau)$ is maximum is defined as $\Delta t$.

$\Delta t$ was calculated from ensemble-averaged wave signals over 50 signals in each direction. The measurements were repeated 100 times for each incident angle and velocity to obtain 100 data. The plots in Fig. 8 represent the averaged $\Delta t$ for 100 data. The error bars represent the variation of the results. The reference lines were calculated from Eqs. (1), (2), and (4) with the refraction angle based on the Lamb wave, which is discussed in the next section. It is well known that the exponent $n$ in Eq. (3) depends on Reynolds number, and $n=7,8$ and 8.3 were used for $V=5.8,12$ and $20 \mathrm{~m} / \mathrm{s}$, respectively (Zhang et al., 2019). $\Delta t$ are in good agreement with the reference values. The uncertainty is related to the signal intensity, as shown in Fig. 6 . The error decreases with increasing signal intensities. 


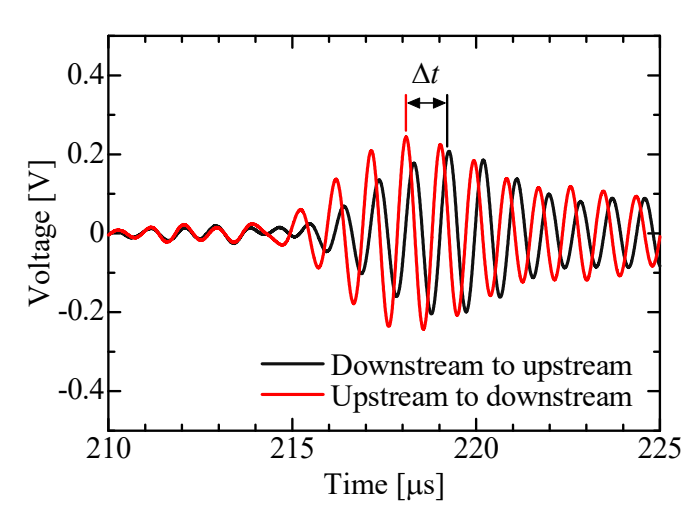

Fig. 7 Target signals in steam flow $(V=12 \mathrm{~m} / \mathrm{s})$ for $80 \mathrm{~A}$ pipe. Transit time of the target signals changed based on the flow velocity. The transit time difference of the target signals is defined as $\Delta t$.

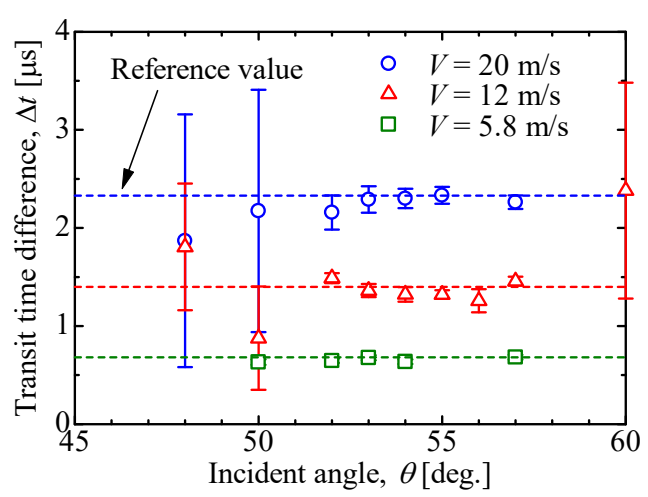

Fig. 8 Effect of incident angle on the transit time difference in steam flow. The color represents each flow velocity. The symbols are plotted as ensemble averaged values over 100 data. The error bar resembles the variance of the data. The dashed lines are reference values for each condition.

\section{Discussion}

The speed of sound of longitudinal waves in polyetherimide is $2450 \mathrm{~m} / \mathrm{s}$, and of shear waves in carbon steel is 3141 $\mathrm{m} / \mathrm{s}$. Therefore, the critical angle between longitudinal waves in the wedge and the shear wave in the pipe is $51^{\circ}$ and is calculated according to Snell's law. Most of our results took the highest intensity beyond the critical angle. The propagated ultrasound wave in the pipe wall was not a body wave like the longitudinal and shear waves. The thickness of a plate is the same order or thinner than the ultrasonic wavelength; ultrasound propagates similarly to plate waves such as Lamb waves. The Lamb wave modes change according to the velocity dispersion from the Rayleigh-Lamb frequency equations and depend on the speed of sound, thickness of the plates, and ultrasound frequency (Fan et al., 2018).

Figure 9 shows the dispersion curves of the phase velocity and the critical angle $\theta_{\mathrm{c}}$ for carbon steel. $S_{n}$ and $A_{n}$ represent the $n$-order of symmetric and antisymmetric modes, respectively. The Lamb wave in each mode can be fully

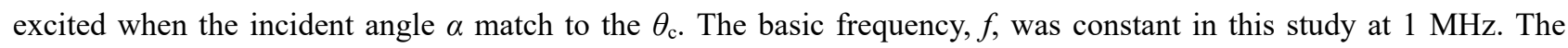
frequency-thickness product, $f d$, depends on the thickness of the pipe wall $d$. In Fig. 9, $f d$ for SGP 25A, 50A, and 80A pipes are indicated as dashed lines. Several Lamb wave modes may occur in each pipe.

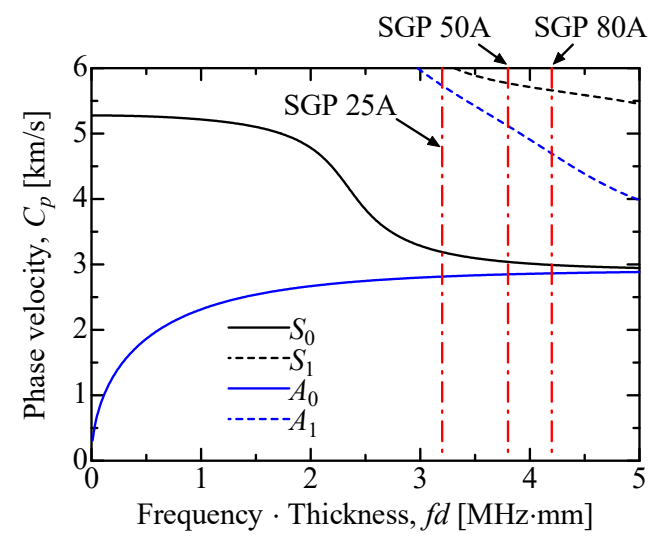

(a) Phase velocity

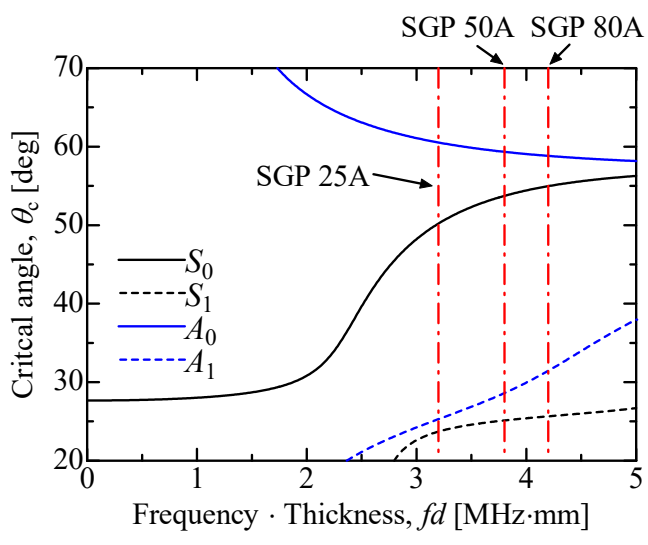

(b) Critical angle in each mode

Fig. 9 Dispersion curves for carbon steel pipe and polyetherimide wedge. Each line represents the Lamb wave mode. The vertical dashed lines represent the frequency-thickness products in each pipe at a constant ultrasonic frequency of 1 MHz.

In $S_{0}$ mode, $\theta_{\mathrm{c}}$ is $50.2^{\circ}$ for SGP $25 \mathrm{~A}, \theta_{\mathrm{c}}$ is $53.7^{\circ}$ for $50 \mathrm{~A}$, and $\theta_{\mathrm{c}}$ is $54.9^{\circ}$ for $80 \mathrm{~A}$. In $A_{0}$ mode, $\theta_{\mathrm{c}}$ is $60.5^{\circ}$ for $\mathrm{SGP}$ $25 \mathrm{~A}, \theta_{\mathrm{c}}$ is $59.3^{\circ}$ for $50 \mathrm{~A}$, and $\theta_{\mathrm{c}}$ is $58.8^{\circ}$ for $80 \mathrm{~A}$. The appropriate incident angle for SGP $80 \mathrm{~A}$ was approximately $54^{\circ}$ 
in both experimental with either nitrogen or steam flow. They were in good agreement with the $\theta_{\mathrm{c}}$ in $S_{0}$ mode. The $S_{0}$ mode had the closest $\theta_{\mathrm{c}}$, including in the cases of stationary nitrogen in the 25A and 50A pipes. Signal intensity at incident angle of $57^{\circ}$ was slightly high for 50A. The angle is close to the $\theta_{\mathrm{c}}$ in $A_{0}$ mode. Therefore, the Lamb wave in $A_{0}$ mode may propagated with large intensity. The intensity distribution for 80A may also include effect of Lamb waves in $A_{0}$ and $S_{0}$ mode because $\theta_{\mathrm{c}}$ in $S_{0}$ and $A_{0}$ mode is close values. However, the main component of the ultrasonic propagation in the pipe wall was most likely in $S_{0}$ mode. The experimental results for $25 \mathrm{~A}$ and $50 \mathrm{~A}$ pipes were slightly lower than the $\theta_{\mathrm{c}}$ in $S_{0}$ mode, stemming from the pipe curvature. The dispersion curves in Fig. 9 were calculated from the Rayleigh-Lamb frequency equations in two dimensions. Therefore, the effect of the pipe curvature was neglected, which resulted in an increased difference of $\theta_{c}$ for smaller diameter pipes.

In normal uses, the range of the $f d$ product with smaller dispersion of phase velocity is appropriate for the measurements (Fan et al., 2018) because the change of the phase velocity depends on a smaller wall thickness. For steam flow measurements using SGP pipes, $f d<2 \mathrm{MHz} \cdot \mathrm{mm}$ in $S_{0}$ mode and/or $f d>3 \mathrm{MHz} \cdot \mathrm{mm}$ in $S_{0}$ and $A_{0}$ modes seem to be appropriate. For flow rate measurements, a larger $\theta$ is more appropriate because $\Delta t$ with respect to flow velocity becomes large. Other measurement uncertainties, such as the transducer setting, became larger for lower $\theta$. When $\alpha$ was $54^{\circ}, \theta$ was $9.5^{\circ}$, and $\theta$ decreased with $\alpha$ based on Snell's law. Therefore, $f d>3 \mathrm{MHz} \cdot \mathrm{mm}$ should be selected for the measurements. Based on the dispersion curves, $\theta_{\mathrm{c}}$ at approximately $60^{\circ}$ in $A_{0}$ mode was the most appropriate for measurements. However, our results indicated that the intensities of the ultrasound signal were low around $\alpha=60^{\circ}$. Therefore, the incident angle of the ultrasound should be selected for $f d>3 \mathrm{MHz} \cdot \mathrm{mm}$ in $S_{0} \mathrm{mode}$ for steam flow measurement.

If $\alpha$ is set at approximately $\theta_{\mathrm{c}}$ in $S_{0}$ mode, the intensity of the target signal was good and $\Delta t$ could be obtained as confirmed from Fig. 8. For a large difference between $\alpha$ and $\theta_{\mathrm{c}}$, the measurement uncertainty increased. Normalized signal intensity of more than 0.25 was required to compare the results with that of intensity in Fig. 6 , which relates to the SNR. The noise signals change with elapsed time after ultrasound emission because the noise is caused mainly by the guided wave. As confirmed by Fig. 4, the guided wave changed with the elapsed time. Therefore, the noise intensity was defined before and after the target signal and evaluated at 0.09 for steam flow in SGP 80A. This range of $\alpha$ was approximately $54^{\circ} \pm 3^{\circ}$. Thus, SNR needs to exceed $9 \mathrm{~dB}$ for accurate measurements. A variable angle ultrasonic transducer is normally of lower sensitivity resulting in lower SNR in comparison to a fixed angle (normal) transducer. Therefore, a fixed angle ultrasonic transducer is normally used. A fixed angle of $54^{\circ}$ transducers may be used in the range of $51<\theta_{\mathrm{c}}<57^{\circ}$. The transducer can be used not only for $80 \mathrm{~A}$ pipe, but also from $50 \mathrm{~A}$ to $150 \mathrm{~A}(\mathrm{fd}=5 \mathrm{MHz} \cdot \mathrm{mm}$, $\theta_{\mathrm{c}}=53.6^{\circ}$ ) etc., because the dispersion curve of $\theta_{\mathrm{c}}$ in $S_{0}$ mode is almost flat at $f d>4 \mathrm{MHz} \cdot \mathrm{mm}$. The adjustment of the incident angle becomes more important with increases in the steam velocity. If the incident angle is appropriately selected for the measurement pipe, the steam velocity can be correctly obtained at steam velocity of $20 \mathrm{~m} / \mathrm{s}$.

\section{Conclusions}

In order to determine the appropriate incident angle for the clamp-on ultrasonic flowmeter to measure steam flow rates, transmitted ultrasonic intensities were measured by changing the ultrasonic incident angle with three pipes (SGP 25A, SGP 50A and SGP 80A) of different diameters made of carbon steel that are generally used for the steam flow.

The incident angle with the highest signal intensity depended on the pipe wall thickness. The incident angles were in good agreement with the critical angle of zero-order Lamb wave in symmetric mode. However, these were slightly lower for pipes with a small diameter, possibly caused by the pipe curvature. Nitrogen and steam experiments yielded the same results; therefore, the propagated ultrasound is a Lamb wave, which enables the setup to increase the transmitted signal intensity. TOF measurements were carried out at steam flow conditions. The transmitted signal intensity decreased because of the turbulent dissipation with increasing steam flow velocity. The appropriate incident angle settings depend on the wall thickness, particularly for higher steam flow conditions, are required to evaluate the transit time difference between upstream and downstream transducers. A fixed angle of $54^{\circ}$ transducers may be used not only for $80 \mathrm{~A}$ pipe, but also from $50 \mathrm{~A}$ to $150 \mathrm{~A}$ pipes.

\section{References}

Fan, Z., Jiang, W. and Wright, W.M.D., Non-contact ultrasonic gas flow metering using air-coupled leaky Lamb waves, 
Ultrasonics, Vol. 89 (2018), pp.74-83. DOI: 10.1016/j.ultras.2018.04.008

Kurniadi, D. and Trisnobudi, A., A multi-path ultrasonic transit time flow meter using a tomography method for gas flow velocity profile measurement, Particle \& Particle Systems Characterization Vol. 23 (2006), pp. 330-380. DOI: $10.1002 /$ ppsc. 200601067

Lynnworth L.C. and Liu Y., Ultrasonic flowmeters: Half-century progress report, 1955-2005, Ultrasonics, Vol. 44 (2006), pp. e1371-e1378. DOI: 10.1016/j.ultras.2006.05.046

Morita, R., Inada, F., Uchiyama, Y., Umezawa, S., Ishibashi, M. and Funaki, T., Influence of steam wetness on steam flow rate measurement using ultrasonic flow meter, Transactions of the JSME (in Japanese), Vol. 83, No. 849 (2017), p. 16-00526. DOI: 10.1299/transjsme.16-00526

Murakawa, H., Ichimura, S., Sugimoto, K., Asano, H., Umezawa, S. and Sugita, K., Evaluation method of transit time difference for clamp-on ultrasonic flowmeters in two-phase flows, Experimental Thermal and Fluid Science, Vol. 112 (2020), 109957. DOI: 10.1016/j.expthermflusci.2019.109957

Nishiguchi, H., Sawayama, T. and Nagamune, K., A propagation time difference evaluation for a clamp-on ultrasonic flowmeter for low-pressure gas, Japanese Journal of Applied Physics, Vol. 55 (2016), 07KB05. DOI: 10.7567/JJAP.55.07KB05

Ramadas, S.N., Harvey, G. and Tweedie, A., Finite element modelling study to explore the possibilities of ultrasonic gas flow measurement in wet-gas applications, IEEE International Ultrasonics Symposium (2016). DOI: 10.1109/ULTSYM.2016.7728420

REFPROP NIST Standard Reference Database 23 (2018).

Sasaki, H. and Hayashi, T., Development of a clamp-on ultrasonic flowmeter for gas, azbil Technical Review (in Japanese), Vol. April 2015 (2015), pp.63-69.

Sasaki, H., Hayashi, T., Umezawa, S. and Sugita, K., Steam flow measurement in the use of clamp-on method, Transactions of the JSME (in Japanese), Vol. 83, No. 847 (2017), p. 16-00397. DOI: 10.1299/transjsme.16-00397

Shen, J.J.S., A new method of steam quality measurement based on ultrasound, Transactions of the ASME J. Energy Resources Technology, Vol. 121 (1999), pp. 172-175. DOI: 10.1115/1.2795978

Steven, R. and Hall, A., Orifice plate meter wet gas flow performance, Flow Measurement and Instrumentation, Vol. 20 (2009), pp. 141-151. DOI: 10.1016/j.flowmeasinst.2009.07.001

Umezawa, S., Shimada, H., Morita, R., Inada, F., Uchiyama, Y., Ishibashi, M. and Funaki, T, Influence of the wetness on flow rate measurement of wet steam using a vortex flow meter, Transactions of the JSME Series B (in Japanese), Vol. 79, No. 799 (2013), pp. 415-422. DOI: 10.1299/kikaib.79.415

Zhang, H., Guo, C. and Lin, J., Effects of velocity profiles on measuring accuracy of transit-time ultrasonic flowmeter, Applied Sciences, Vol. 9, No. 8 (2019), 1648. DOI: 10.3390/app9081648 